\title{
Correlations of correlations: Secondary autocorrelations in finite harmonic systems
}

\author{
Dan Plyukhin, 1 , and Alex V. Plyukhin², 田 \\ 1 Department of Computer Science, University of Toronto, Toronto, ON, Canada \\ ${ }^{2}$ Department of Mathematics, Saint Anselm College, Manchester, NH, USA
}

(Dated: August 24, 2018)

\begin{abstract}
The momentum or velocity autocorrelation function $C(t)$ for a tagged oscillator in a finite harmonic system decays like that of an infinite system for short times, but exhibits erratic behavior at longer time scales. We introduce the autocorrelation function of the long-time noisy tail of $C(t)$ ("a correlation of the correlation"), which characterizes the distribution of recurrence times. Remarkably, for harmonic systems with same-mass particles this secondary correlation may coincide with the primary correlation $C(t)$ (when both functions are normalized) either exactly, or over a significant initial time interval. When the tagged particle is heavier than the rest, the equality does not hold, correlations shows non-random long-time scale pattern, and higher order correlations converge to the lowest normal mode.

PACS numbers: 05.40.Ca, 05.20.Gg
\end{abstract}

\section{INTRODUCTION}

The theme of fluctuations in finite systems of harmonic oscillators emerges naturally in both application and theory. From a theoretical point of view, the study of the stochastic dynamics of a tagged degree of freedom in finite harmonic systems provides a valuable illustration, and often more than that, of the role of the thermodynamic and weak-coupling limits, ergodicity, thermalization, recurrences, synchronization, and other basic concepts in nonequilibrium phenomena [1 7]. Another relevant area is Langevin dynamics generated by a coupling to a finite harmonic bath(s), and its application to mesoscopic systems and networks; see [9 15].

Being nonergodic, the capability of harmonic systems to illustrate general phenomena in statistical mechanics might seem doubtful at first glance. By means of a canonical transformation a harmonic system of any size can be transformed into a collection of independent oscillators, or normal modes; since the energies of normal modes are the integrals of motion, a single isolated harmonic system does not equilibrate and is not very interesting from the point of view of statistical mechanics.

A more fruitful approach is to consider an ensemble of harmonic systems, assuming that in the past they were in contact with a larger thermal bath in equilibrium at a given temperature, and that the initial normal modes of the ensemble are distributed according to the canonical distribution. Within this framework, one evaluates statistical averages of dynamical variables over the ensemble of the system's initial coordinates rather than over time. Such averages show the transition of the ensemble to thermal equilibrium in the limit of a large number of particles, and thus the nonergodic nature of harmonic systems does not explicitly manifest itself, and for

\footnotetext{
*Electronic address: dplyukhin@cs.utoronto.ca
}

†Electronic address: aplyukhin@anselm.edu most cases is inessential. It should however be stressed that this framework, which is standard for most works on stochastic dynamics of harmonic systems both classical and quantum, assumes a very special type of coupling between the system and the external thermal bath: this coupling justifies the initial conditions for the the system's degrees of freedom, yet is assumed to be sufficiently weak, or completely turned off, as not to affect the system's further dynamics.

The inequivalence for harmonic systems of ensembleand time-averages, together with the almost exclusive exploitation in literature of the former, does not necessarily entail that the latter are inadequate. Rather, we introduce in this paper a new class of time-average correlations (we call these secondary correlations) which characterize recurrences in finite harmonic systems. For systems of same-mass particles, these correlations are shown to be very close, and under certain conditions exactly identical, to the conventional (primary) time correlations defined with ensemble averaging. This implies that for finite nonergodic systems, the use of both ensemble and time averages may give meaningful complementary descriptions, and that correlations with the two types of averaging may be related in some subtle way.

\section{SECONDARY CORRELATIONS}

Consider the temporal autocorrelation function $\langle A(0) A(t)\rangle$ of a dynamical variable $A$ in a finite system of size $L$ - typically, such a function exhibits two distinctive regimes, separated by a crossover time $t_{c}$ of order $L / v$, where $v$ is the speed of signal propagation in the system. For short times $t<t_{c}$, the variable does not feel the presence of the boundaries, and the correlation function decays in a smooth regular way, following the same laws as for an infinitely large system. On the other hand, for longer times $t>t_{c}$ the dynamics of the variable are affected by signals reflected from the boundaries. For long time regimes such as this, rather than decaying smoothly 
the correlation functions may exhibit erratic, apparently noisy, behavior [1, 2].

We illustrate this behavior in Fig. 1 by way of the normalized momentum correlation function for the central particle in a harmonic chain with fixed ends. The Hamiltonian of the system is

$$
H=\frac{1}{2 m} \sum_{i=1}^{N} p_{i}^{2}+\frac{m \omega^{2}}{2} \sum_{i=0}^{N}\left(q_{i}-q_{i+1}\right)^{2},
$$

which describes $N+2$ linearly coupled particles indexed $i=0,1, \ldots, N+1$ with terminal particles fixed, with displacement $q_{0}=q_{N+1}=0$. Assuming $N$ is odd, the middle particle indexed

$$
i_{0}=\frac{N+1}{2}
$$

has normalized $(C(0)=1)$ momentum correlation function

$$
C_{i_{0}}(t)=\frac{1}{\left\langle p_{i_{0}}^{2}(0)\right\rangle}\left\langle p_{i_{0}}(0) p_{i_{0}}(t)\right\rangle=\frac{2}{N+1} \sum_{j=1}^{N}{ }^{\prime} \cos \omega_{j} t
$$

where the prime indicates that the summation is only over odd $j$. In this expression (we outline its derivation in the Appendix), the $\omega_{j}$ terms are frequencies of normal modes

$$
\omega_{j}=2 \omega \sin \frac{\pi j}{2(N+1)}
$$

where $\omega$ is the frequency of a single oscillator, and the average $\langle\cdots\rangle$ is taken over the equilibrium ensemble of initial conditions. For $t<t_{c}$ the correlation function $C_{i}(t)$ is very close to that of an infinite chain, given by the Bessel function

$$
C_{i}(t) \approx C_{\infty}(t)=\lim _{i, N \rightarrow \infty} C_{i}(t)=J_{0}(2 \omega t) .
$$

This can be readily justified by approximating the sum (3) with an integral, and recognizing the latter as the well-known integral representation of $J_{0}(2 \omega t)$, see e.g. 8, 10].

More interesting from the perspective of this paper is the regime $t>t_{c}$ in which the correlation $C_{i}(t)$ becomes irregular, see Fig. 1. It can be shown that the function $C_{i}(t)$ given by (3) belongs to the class of almost periodic functions: any value $c$ which the function achieves once is achieved again, infinitely many times. Traditionally, such functions are characterized by the average frequency with which they return to $c$, or by the reciprocal, i.e. the mean recurrence time $\tau(c)$. For correlations of type (3) with large $N$, the famous result for the recurrence time, first obtained by Kac [4] (see also [1, 2, 5]),

$$
\tau(c) \sim e^{N c^{2}}
$$

implies that recurrences of order $c \sim N^{0}$ or larger are exponentially rare. This result resolves, or rather (being derived for a model system) shows the direction of resolution for the paradoxes of irreversibility [1].

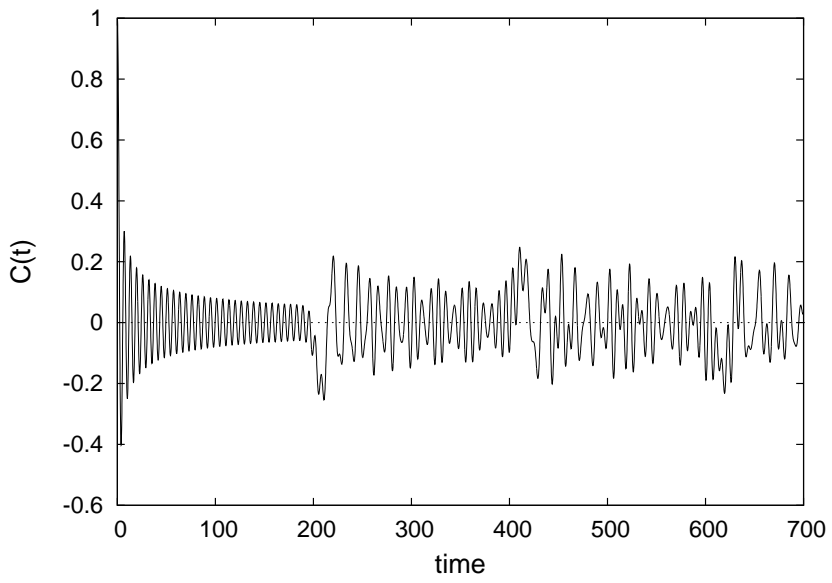

FIG. 1: The normalized momentum correlation function $C_{i}(t)$ for the central particle $\left(i=i_{0}=51\right)$ of the harmonic chain with fixed ends with Hamiltonian (1) with $N=101$, given by Eq. (3). The time unit is $1 / 2 \omega$. The time of the crossover from the regular dissipation to "stochastic" regimes is $t_{c} \approx$ 200.

In this paper we propose to characterize the irregular part of the function $C_{i}(t)$ in another way, which is more in the spirit of nonequilibrium statistical mechanics than the mathematics of almost periodic functions. Namely, observing that for large $t$ the correlation function $C_{i}(t)$ appears to behave like stationary noise, we are encouraged to characterize it by a new correlation function

$$
D_{i}(t)=\frac{1}{\left\langle C_{i}^{2}(\tau)\right\rangle_{\tau}}\left\langle C_{i}(\tau) C_{i}(\tau+t)\right\rangle_{\tau}
$$

defined with the time average

$$
\langle\ldots\rangle_{\tau}=\lim _{T \rightarrow \infty} \frac{1}{T} \int_{0}^{T}(\ldots) d \tau
$$

Since we are only interested in the interval $t>t_{c}$ when $C_{i}(t)$ behaves irregularly, one might prefer to set the lower integration limit in definition (8) to $t_{c}$ instead of zero. However this would only be an unnecessary complication, as the limit $T \rightarrow \infty$ makes the two definitions numerically equivalent (assuming always that the integral from 0 to $t_{c}$ converges).

We shall refer to $D_{i}(t)$, defined by relations (7) and (8), as the secondary correlation function, and call $C_{i}(t)$ the primary one. We would like to promote the secondary correlation $D_{i}(t)$ as a meaningful statistical tool for characterizing the distribution of recurrences times in a system of finite size. Such information is not contained in the Kac formula (6) for the average recurrence time $\tau$, so the two functions $\tau(c)$ and $D_{i}(t)$ do not duplicate each other but describe recurrences in complementary ways. 


\section{RELATION TO PRIMARY CORRELATIONS}

Since the primary and secondary correlations $C_{i}(t)$ and $D_{i}(t)$ characterize recurrences at different levels and are defined using different types of averaging (over ensemble and time, respectively), the existence of any specific relation between them is perhaps a priori unexpected. Yet a simple numerical experiment with Eqs. (1-6) suggests, for the middle atom of a chain with fixed ends, the equality

$$
C_{i_{0}}(t)=D_{i_{0}}(t)
$$

Closer scrutiny reveals that the equality is exact and holds for any $t$, such that the secondary correlation completely repeats the structure of the primary one for both regular $\left(t<t_{c}\right)$ and noisy $\left(t>t_{c}\right)$ domains and has the same crossover time $t_{c}$. The proof follows immediately from the relation

$$
\left\langle\cos \omega_{j} \tau \cos \omega_{j^{\prime}}(\tau+t)\right\rangle_{\tau}=\frac{\delta_{j j^{\prime}}}{2} \cos \omega_{j} t
$$

which holds for an arbitrary spectrum of (nonzero) normal mode frequencies $\left\{\omega_{j}\right\}$ and can be verified by direct evaluation (with the help of L'Hospital's rule). For $t=0$ this may further be reduced to the familiar orthogonality relation for the Fourier basis, and thus can be considered a generalized form of the latter. From (3) and (10) one obtains for the non-normalized secondary correlation

$$
\begin{aligned}
& \left\langle C_{i_{0}}(\tau) C_{i_{0}}(\tau+t)\right\rangle_{\tau}= \\
& \left(\frac{2}{N+1}\right)^{2} \sum_{j, k=1}^{N}\left\langle\cos \omega_{j} \tau \cos \omega_{k}(\tau+t)\right\rangle_{\tau}= \\
& \frac{1}{2}\left(\frac{2}{N+1}\right)^{2} \sum_{j=1}^{N} \cos \omega_{j} t
\end{aligned}
$$

Normalizing this function to unity at $t=0$ by dividing it by

$$
\left\langle C_{i_{0}}^{2}(\tau)\right\rangle_{\tau}=\frac{1}{2}\left(\frac{2}{N+1}\right)^{2} \frac{N+1}{2}=\frac{1}{N+1},
$$

one obtains the normalized secondary correlation

$$
D_{i_{0}}(t)=\frac{\left\langle C_{i_{0}}(\tau) C_{i_{0}}(\tau+t)\right\rangle_{\tau}}{\left\langle C_{i_{0}}^{2}(\tau)\right\rangle_{\tau}}=\frac{2}{N+1} \sum_{j=1}^{N} \cos \omega_{j} t
$$

which coincides with the primary correlation $C_{i_{0}}(t)$, Eq.(3).

One may observe that for the above derivation it is essential that the primary correlation $C_{i}(t)$ takes the form of a superposition of cosines with equal weights, as in Eq. (3). In general this, of course, is not the case. For example, for a chain with fixed ends described by the Hamiltonian (11), the normalized momentum correlation

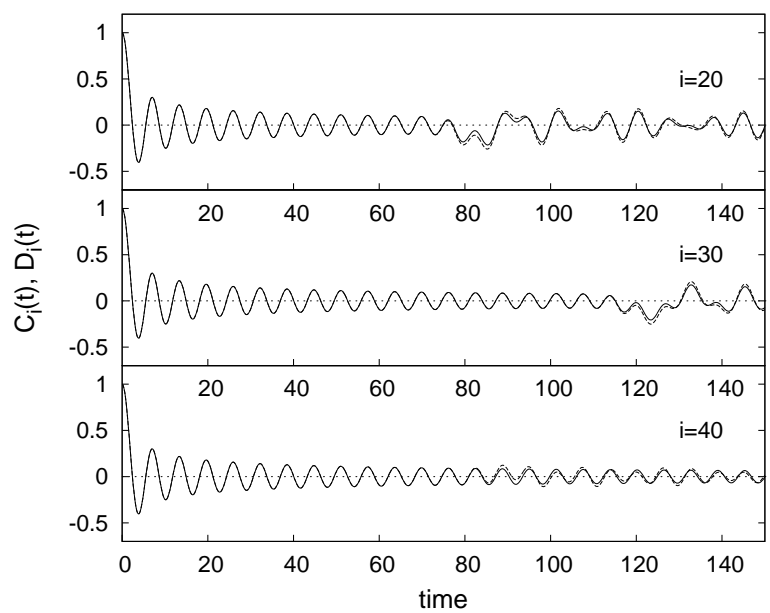

FIG. 2: The primary momentum correlation function $C_{i}(t)$ given by Eq. (14) (solid line) and secondary correlation function $D_{i}(t)$ given by Eq. (17) (dashed line) for the harmonic chain with Hamiltonian (1) with $N=101$ for particles $i=20$ (top), $i=30$ (middle), and $i=40$ (bottom). The difference between $C_{i}(t)$ and $D_{i}(t)$ becomes noticeable for $t>t_{0}$ where $t_{0}$ depends on $i$ non-monotonically: $t_{0} \approx 80,120,90$, from top to the bottom.

function for particle with arbitrary index $i$ has the form (see Appendix)

$$
C_{i}(t)=\sum_{j=1}^{N} A_{i j}^{2} \cos \omega_{j} t, \quad A_{i j}=\sqrt{\frac{2}{N+1}} \sin \frac{\pi i j}{N+1} .
$$

For the middle particle $i=i_{0}=(N+1) / 2$ this is reduced to (3), whereas for the other particles normal modes enter the expression (14) with different amplitudes $A_{i j}^{2}$. As one can immediately verify, the exact equality of primary and secondary correlations does not hold in these cases. An important example when this equality does hold for any particle is a harmonic chain with periodic boundary conditions. In this case the momentum correlation for each particle is a superposition of equally weighted normal modes [1, 2]

$$
C_{i}(t)=\frac{1}{N} \sum_{j=0}^{N-1} \cos \omega_{j} t, \quad \omega_{j}=2 \omega \sin \left(\frac{\pi j}{N}\right),
$$

and repetition of the above derivation leads again to the exact equality $C_{i}(t)=D_{i}(t)$ for any particle of the system.

So far, even with the above examples of its validity, the equality of primary and secondary correlations may appear as no more than a curious coincidence. However, further numerical exercises reveal that even when equality does not hold exactly, it remains a very good approximation for the initial time interval $t<t_{0}$, see Fig. 2. The duration of this interval, $t_{0}$, is found to depend non-monotonically on particle position $i$, and for any $i$ be equal or shorter than the crossover time, $t_{0} \leq t_{c}$. 
Respectively, for $t<t_{0}$ both primary and secondary correlations coincide with the primary correlation for the infinite chain,

$$
D_{i}(t)=C_{i}(t)=C_{\infty}(t)=J_{0}(2 \omega t), \quad t<t_{0} \leq t_{c} .
$$

The proof of the approximate equality (16) can be carried out as follows. From the expression (14) for $C_{i}(t)$ and the definition (77) for $D_{i}$, and using the relation (10), one gets

$$
D_{i}(t)=\frac{1}{\sum_{j=1}^{N} A_{i j}^{4}} \sum_{j=1}^{N} A_{i j}^{4} \cos \omega_{j} t
$$

or taking into account the expression (4) for normal mode frequencies

$$
D_{i}(t)=\frac{1}{\sum_{j=1}^{N} A_{i j}^{4}} \sum_{j=1}^{N} A_{i j}^{4} \cos \left[2 \omega t \sin \left(\frac{\pi j}{2(N+1)}\right)\right] .
$$

Recognizing here the generating function for Bessel functions

$$
\cos (x \sin \theta)=J_{0}(x)+2 \sum_{k=1}^{\infty} J_{2 k}(x) \cos (2 k \theta),
$$

$D_{i}(t)$ can be written as a superposition of Bessel functions

$$
D_{i}(t)=J_{0}(2 \omega t)+\sum_{k=1}^{\infty} S_{i k} J_{2 k}(2 \omega t)
$$

with coefficients

$$
S_{i k}=\frac{2}{\sum_{j=1}^{N} A_{i j}^{4}} \sum_{j=1}^{N} A_{i j}^{4} \cos \left(\frac{\pi j k}{N+1}\right) .
$$

A simple analysis of this expression shows that given $i$, the coefficients $S_{i k}$ are nonzero only for five sets of $k$ :

$$
S_{i k}=\left\{\begin{array}{cl}
2, & k=2(N+1) s \\
-4 / 3, & k=2(N+1) s-2 i \\
-4 / 3, & k=2(N+1)(s-1)+2 i \\
1 / 3, & k=2(N+1) s-4 i \\
1 / 3, & k=2(N+1)(s-1)+4 i \\
0, & \text { otherwise }
\end{array}\right.
$$

where $s=1,2,3, \ldots$ Note that this expression is invariant under the transformations $i \rightarrow(N+1)-i$, reflecting the symmetry of the left and right sides of the chain. One can observe that for large $N$ and $i$ not too close to the end or to the middle of the chain the coefficients $S_{i k}$ are nonzero only for large indices $k$. For instance, for the chain with $N=101$ and the particle $i=20$, coefficients

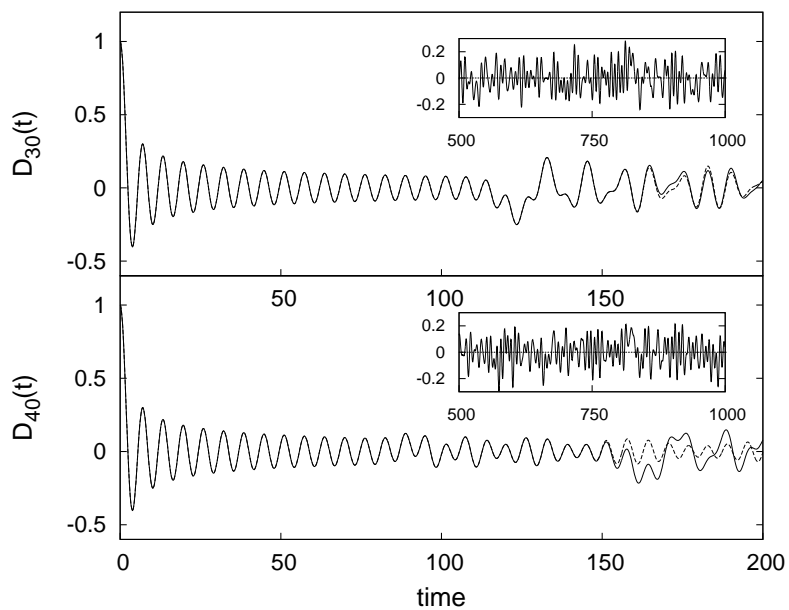

FIG. 3: The secondary correlation functions $D_{30}(t)$ (top) and $D_{40}(t)$ (bottom) according to the exact expression (17) (solid lines) and the approximation (23) (dashed lines). The insets show apparently random behavior of $D_{30}(t)$ and $D_{40}(t)$ at longer times.

$S_{i k}$ are nonzero only for $k=40,80,124, \ldots$ As a result, for $t$ not too large in the expression (20), the dominating contribution comes from the first term $J_{0}(2 \omega t)$, while the corrections given by the sum $\sum_{k=1}^{\infty} S_{i k} J_{2 k}(2 \omega t)$ involve Bessel functions of large orders which are negligibly small for a significant time interval $t<t_{0}[16]$.

The above consideration not only justifies the equality $D_{i}(t)=C_{i}(t)=J_{0}(2 \omega t)$ for $t<t_{0}$, but also accounts for a curious non-monotonic dependence of $t_{0}$ on the tagged particle index $i$, which we noticed empirically in Fig. 2. For example, according to (22), for $N=101$ and particles $i=20,30,40$ the minimal indices $k$ for which $S_{i k}$ takes nonzero values $(-4 / 3,-4 / 3,1 / 3)$ are $k=40,60,44$, respectively. Then keeping only the leading and first correction terms in the exact expression (20), one gets

$$
\begin{aligned}
& D_{20}(t)=J_{0}(2 \omega t)-\frac{4}{3} J_{80}(2 \omega t), \\
& D_{30}(t)=J_{0}(2 \omega t)-\frac{4}{3} J_{120}(2 \omega t), \\
& D_{40}(t)=J_{0}(2 \omega t)+\frac{1}{3} J_{88}(2 \omega t) .
\end{aligned}
$$

One can verify that these approximations describe the initial deviation of $D_{i}(t)$ from $C_{\infty}(t)=J_{0}(2 \omega t)$ very well indeed (Fig. 3 shows this for particle $i=30$ and $i=40$ ). Since for small arguments $J_{i}(x)$ decreases with order $i$, it is clear from (23) that the second correction terms for particles $i=20,40$ involve Bessel functions of smaller orders, and thus become essential at earlier times than for particle $i=30$.

If one applies a similar analysis to the primary correlations $C_{i}(t)$, Eq. (14), one gets a familiar approximate relation for the left side of the chain [5, 8, 10]

$$
C_{i}(t)=J_{0}(2 \omega t)-J_{4 i}(2 \omega t) .
$$


Here, in contrast to corresponding relations (23) for $D_{i}(t)$, the order of the second Bessel function, which describes effects of finite size, increases monotonically (linearly) with particle index $i$, and so does the crossover time $t_{c}$.

In order to study the dependence of the characteristic time $t_{0}$, during which $C_{i}(t)=D_{i}(t)$, on $i$ in a more quantitative way, let us consider the function

$$
\delta_{i}(t)=C_{i}(t)-D_{i}(t),
$$

which is zero when the two correlations coincide for $t<$ $t_{0}$ and fluctuates at longer times. For a given $i$, let us define $t_{0}$ somewhat arbitrarily as the time at which $\delta_{i}(t)$ reaches its first local minimum or maximum; see Fig. 4(a). Similarly, we can define the crossover time $t_{c}$ as the moment when the function

$$
\Delta_{i}(t)=C_{i}(t)-C_{\infty}(t)
$$

has its first local extremum, recalling that $C_{\infty}(t)=$ $J_{0}(2 \omega)$ is the correlation in an infinite system. Using these definitions, we record observations of $t_{0}$ and $t_{c}$ for $N=101$ in Fig. 4(b), as a function of particle index $i$. Whereas $t_{c}$ increases linearly as we approach the central particle, $t_{0}$ coincides with $t_{c}$ for $i<i_{1}=34$ and linearly decreases for $i>i_{1}$. As we already know, the primary and secondary correlations coincide for the middle particle $i_{0}=51$, so $\delta_{i_{0}}(t)$ is identically 0 and $t_{0}$ diverges here. Somewhat unexpectedly, we find that $t_{0}$ also diverges, i.e. $C_{i}(t)=D_{i}(t)$ identically, for $i_{1}=34$ (and of course the symmetric case $\left.i_{2}=(N+1)-i_{1}=68\right)$. Therefore it would appear that $t_{0}(i)$ diverges whenever it changes from increasing to decreasing, or vice versa. Further calculations for different $N$ show that in general the exact equality $C_{i}(t)=D_{i}(t)$ holds for particles with indices

$$
i_{0}=\frac{N+1}{2}, \quad i_{1}=\frac{N+1}{3}, \quad i_{2}=\frac{2(N+1)}{3}
$$

provided of course that these expressions are integers. For $N=101$ there are three such particles $\left(i_{0}=51, i_{1}=\right.$ $\left.34, i_{2}=68\right)$, two for $N=200\left(i_{1}=67, i_{2}=134\right)$, and none for $N=100$.

Let us show that this phenomenon is readily accounted for with Eqs.(20)-(22) for the secondary correlation $D_{i}(t)$. First, from inspecting (22) one might observe that for $i<i_{0}$ the minimum $k$ for which $S_{i k}$ is non-zero is $k=2 i$ and comes from the set $k=2(N+1)(s-1)+2 i$ with $s=1$. This yields the approximation

$$
D_{i}(t)=J_{0}(2 \omega t)-\frac{4}{3} J_{4 i}(2 \omega t),
$$

which we already used for $D_{20}(t)$ and $D_{30}(t)$ in (23). It differs from the corresponding approximation (24) for $C_{i}(t)$ only by the factor $4 / 3$ in the second term. Then, from (28) and (24), the difference functions defined above by relations (25) and (26) take the form

$$
\delta_{i}(t)=\frac{1}{3} J_{4 i}(2 \omega t), \quad \Delta_{i}(t)=-J_{4 i}(2 \omega t) .
$$
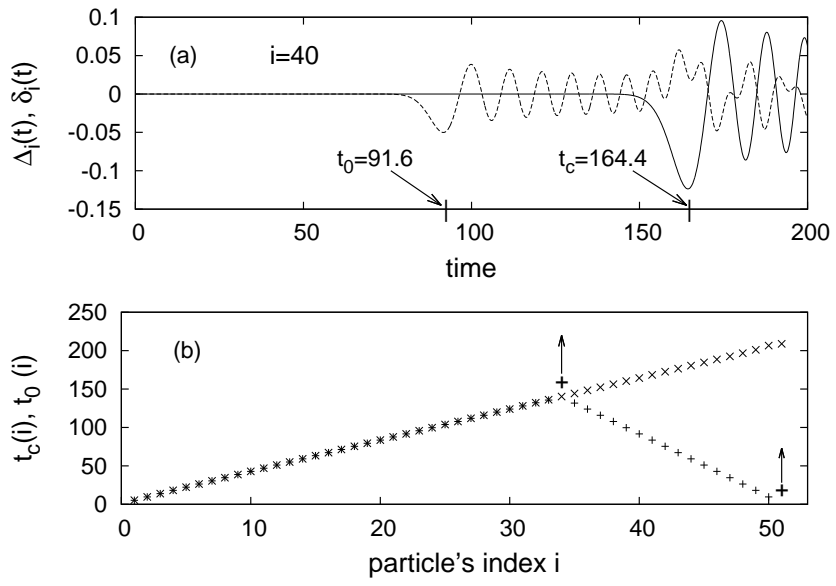

FIG. 4: Top plot (a): The functions $\Delta_{i}(t)=C_{i}(t)-J_{0}(2 \omega t)$ (solid line) and $\delta_{i}(t)=C_{i}(t)-D_{i}(t)$ (dashed line) for particle $i=40$. The characteristic times $t_{c}$ and $t_{0}$ are defined as times at which $\Delta_{i}(t)$ and $\delta_{i}(t)$, respectively, have their first local extremum. Bottom plot (b): The characteristic times $t_{c}$ $(\times)$ and $t_{0}(+)$ for particles with indices $i \leq 51$ for the left side of the chain described by Hamiltonian (1) with $N=101$. For particles $i_{0}=51$ and $i_{1}=34$ the time $t_{0}$ diverges (illustrated by an arrow pointing upward), indicating the exact equality $C_{i}(t)=D_{i}(t)$.

Since these two functions have local extrema at the same time, by definition we have $t_{0}=t_{c}$. Furthermore, since the position of the first maximum of the Bessel function $J_{i}(t)$ increases approximately linear with $i$ [17], Eq. (29) explains the equality of the characteristic times $t_{c}(i)=$ $t_{0}(i)$ and their linear increase for $i<i_{0}$ in Fig. 4(b).

As $i$ gets larger still, one observes from (22) that a minimal $k$ for which $S_{i k} \neq 0$ is $k=2(N+1)-4 i$ and comes from the set $k=2(N+1) s-4 i$ with $s=1$. In this case for $D_{i}(t)$, instead of (28), we have another approximation

$$
\begin{aligned}
D_{i}(t) & =J_{0}(2 \omega t)+\frac{1}{3} J_{\alpha}(2 \omega t), \\
\alpha & =4(N+1)-8 i,
\end{aligned}
$$

which we already used for $D_{40}(t)$ in (23). Since the primary correlation $C_{i}(t)$ is still given by (24), the difference function $\delta_{i}(t)=C_{i}(t)-D_{i}(t)$ in this case reads

$$
\delta_{i}(t)=-\frac{1}{3} J_{\alpha}(2 \omega t)-J_{4 i}(2 \omega t) \approx-\frac{1}{3} J_{\alpha}(2 \omega t) .
$$

The position of its first extremum increases approximately linearly with $\alpha$ [17] and, as follows from (30), linearly decreases with $i$. This explains the behavior of $t_{0}(i)$ for $i>i_{1}$ in Fig. 4(b).

The transition of $t_{0}(i)$ from a positive to a negative slope (over the domain $[0,51]$ ) occurs at $i=i_{1}$, for which $k_{2}=2(N+1)-4 i$ (the minimal value of the set $k=$ $2(N+1) s-4 i)$ becomes less than or equal to $k_{1}=2 i$ (the minimal value of the set $k=2(N+1)(s-1)+2 i$ ). 
Then the equality $k_{1}=k_{2}$ gives $i_{1}=(N+1) / 3$, which is consistent with our empirical findings (27).

The exact equality $C_{i}(t)=D_{i}(t)$ for $i$ given by (27) can be readily verified using the following expression for the primary correlations

$$
C_{i}(t)=J_{0}(2 \omega t)+\sum_{k=1}^{\infty} T_{i k} J_{2 k}(2 \omega t),
$$

with coefficients

$$
T_{i k}=2 \sum_{j=1}^{N} A_{i j}^{2} \cos \left(\frac{\pi j k}{N+1}\right) .
$$

These relations are similar to (20) and (21) for $D_{i}(t)$ and can be derived in a similar way [10]. For $i=i_{0}, i_{1}, i_{2}$ given by (27), one can verify directly from (33) and (21) that $S_{i k}=T_{i k}$ for any $k$. Then the comparison of (32) and (20) gives for those values of $i$ the exact equality $C_{i}(t)=D_{i}(t)$.

\section{HEAVY IMPURITY PROBLEM}

So far we have discussed finite harmonic systems of similar particles. If a tagged particle is heavier than the rest, it turns out that the equality of primary and secondary correlations, $C(t)$ and $D(t)$, does not hold. Though structurally similar - $D(t)$ looks like a coarsegrained copy of $C(t)$ - the two correlations are quite distinctive on any time scale; see Fig. 5. In particular, the approximation of exponential relaxation for $t<t_{c}$, while good for $C(t)$, is noticeably worse for $D(t)$. Another observation is that for $t \gg t_{c}$ both correlations, being apparently random on a short time scale, show on a larger scale a noisy yet periodically repeating pattern; see the bottom plot in Fig. 5. This feature, absent in systems of equal-mass particles, is made all the more obvious when considering higher order correlation functions $C_{k}(t)$, defined recursively as

$$
C_{k+1}(t)=\frac{\left\langle C_{k}(\tau) C_{k}(\tau+t)\right\rangle_{\tau}}{\left\langle C_{k}^{2}(\tau)\right\rangle_{\tau}}
$$

assuming new notations for $C(t)=C_{1}(t)$ and $D(t)=$ $C_{2}(t)$. (In this section we use the notation $C_{k}(t)$ with a subscript referring to the correlation order, rather than to the index of a particle). For the heavy impurity problem one finds that as the order $k$ increases the apparent randomness of correlations $C_{k}(t)$ on the time scale $t>\tau_{c}$ quickly diminishes, and higher correlations converge to the normal mode with the lowest eigenfrequency $\Omega_{1}$ :

$$
C_{k}(t) \rightarrow \cos \left(\Omega_{1} t\right)
$$

see Fig. 6. Below we outline a theoretical framework underlying these empirical observations.

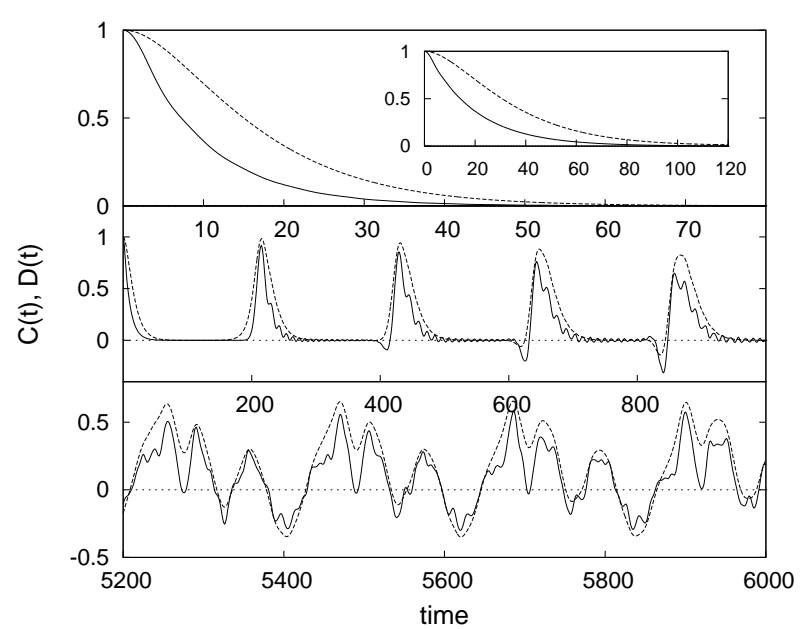

FIG. 5: The primary (solid line) and secondary (dashed line) momentum correlation functions, $C(t)$ and $D(t)$, for the heavy impurity problem described by Hamiltonian (36) with mass ratio $\mu=m / M=0.1$ and $N=50$. The top, middle, and bottom figures show the evolution of the correlations on short $\left(t<t_{c}\right)$, intermediate $\left(t \sim t_{c}\right)$, and long $\left(t \gg t_{c}\right)$ time scales. The inset shows correlations on the short time scale for an impurity that is twice as heavy; $\mu=0.05$. Time is in units of $1 / 2 \omega$.

Consider a cyclic chain of $2 N$ particles of mass $m$ and an impurity of mass $M>m$ described by the Hamiltonian

$$
\begin{aligned}
H & =\frac{P^{2}}{2 M}+\sum_{i=1}^{2 N} \frac{p_{i}^{2}}{2 m}+\frac{m \omega^{2}}{2} \sum_{i=1}^{2 N-1}\left(q_{i}-q_{i+1}\right)^{2} \\
& +\frac{m \omega^{2}}{2}\left[\left(Q-q_{1}\right)^{2}+\left(Q-q_{2 N}\right)^{2}\right],
\end{aligned}
$$

where $P$ and $Q$ are the momentum and coordinates of the impurity. Using a diagonalization method similar to that described in the Appendix (see [3] for details), one can show that the normalized momentum correlation function for the impurity $C(t)=\langle P(0) P(t)\rangle /\left\langle P^{2}(0)\right\rangle$ is again an almost periodic function, now of the form

$$
C(t) \equiv C_{1}(t)=\sum_{j=0,1,3, \cdots}^{2 N-1} A_{j} \cos \Omega_{j} t .
$$

The amplitudes $A_{j}$ in this expression are given by

$$
A_{j}=\left\{1+\sum_{i=1,3, \ldots}^{2 N-1} \frac{\epsilon_{i}^{2}}{\left(\Omega_{j}^{2}-\omega_{i}^{2}\right)^{2}}\right\}^{-1} .
$$

where

$$
\begin{aligned}
\omega_{i} & =2 \omega \sin \frac{i \pi}{2(2 N+1)} \\
\epsilon_{i} & =-2 \mu^{\frac{1}{2}} \omega^{2}\left(\frac{2}{2 N+1}\right)^{\frac{1}{2}} \sin \frac{i \pi}{2 N+1},
\end{aligned}
$$




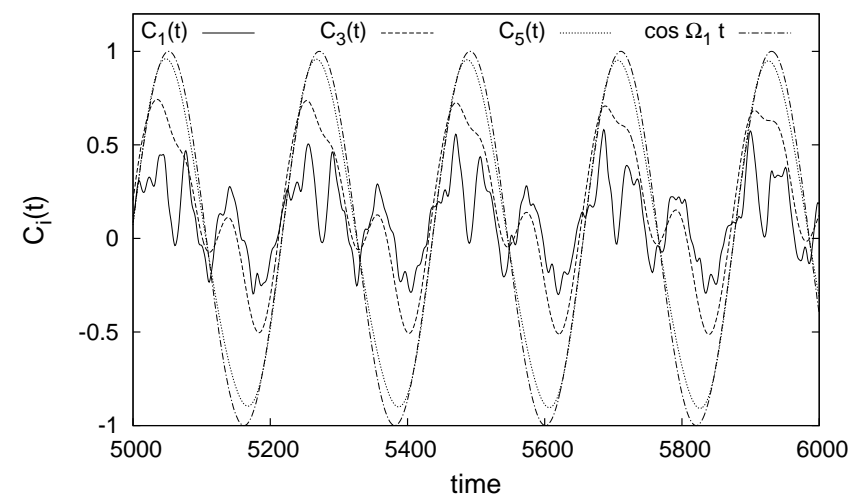

FIG. 6: The primary correlation $C_{1}(t)$ (solid line), and two higher correlations $C_{3}(t)$ (dashed line) and $C_{5}(t)$ (dotted line), defined by Eq. (34), at long time $t \gg t_{c}$ for the heavy impurity problem with $\mu=0.1$ and $N=50$. Higher correlations converge to the lowest normal mode $\cos \Omega_{1} t$ (dot dashed line).

and $\mu=m / M$ is the mass ratio.

Due to the system's symmetry only the modes with zero and odd indices contribute to the superposition (37). Their frequencies $\Omega_{j}(j=0,1,3, \cdots 2 N-1)$ for $M \neq m$ cannot be expressed in closed form and must be evaluated as roots of the secular equation [3]

$$
G(z)=z^{2}-2 \mu \omega^{2}-\sum_{i=1,3, \cdots}^{2 N-1} \frac{\epsilon_{i}^{2}}{z^{2}-\omega_{i}^{2}}=0 .
$$

This transcendental equation has $N+1$ solutions $z=$ $\Omega_{j}, j=0,1,3, \cdots 2 N-1$. It can be verified that one solution is the zero frequency $\Omega_{0}=0$, which reflects the translational invariance of the system. The remaining $N$ nonzero roots $\Omega_{1}, \Omega_{3}, \ldots \Omega_{2 N-1}$ lie in the interval $(0,2 \omega)$ and must be evaluated numerically.

With the set of eigenfrequencies $\Omega_{j}$ found, one may calculate the amplitudes $A_{j}$ with (38) and evaluate the primary correlation $C(t)$ by carrying out the summation in (37). Then, using (10), for the secondary correlation $D(t)$ one obtains

$$
D(t)=C_{2}(t)=c_{2}\left\{A_{0}^{2}+\frac{1}{2} \sum_{j=1,3, \cdots}^{2 N-1} A_{j}^{2} \cos \Omega_{j} t\right\}
$$

with normalization coefficient

$$
c_{2}=\left(A_{0}^{2}+\frac{1}{2} \sum_{j=1,3, \cdots}^{2 N-1} A_{j}^{2}\right)^{-1} .
$$

Fig. 5 presents $C(t)$ and $D(t)$, calculated with Eqs. (37) and (41), for $\mu=0.1$ and $N=50$. In a similar manner, one can obtain the expression for order- $k$ correlations from (34)

$$
C_{k}(t)=c_{k}\left\{A_{0}^{\alpha_{k}}+\sum_{j=1,3, \cdots}^{2 N-1}\left(\frac{A_{j}}{\sqrt{2}}\right)^{\alpha_{k}} \cos \Omega_{j} t\right\}
$$

with powers $\alpha_{k}=2^{k-1}$ and normalization coefficient

$$
c_{k}=\left\{A_{0}^{\alpha_{k}}+\sum_{j=1,3, \cdots}^{2 N-1}\left(\frac{A_{j}}{\sqrt{2}}\right)^{\alpha_{k}}\right\}^{-1} .
$$

While expression (43) is a superposition of $N+1$ modes, one can observe that for larger $k$ the main contribution comes from the mode with eigenfrequency $\Omega_{1}$, such that $C_{k}(t) \approx \cos \Omega_{1} t$. This can be accounted for by noticing that the sequence of coefficients $\left\{A_{j}\right\}$ has $A_{1}$ as its maximum element and is monotonically decreasing for $j>0$. For example, for $\mu=0.1$ and $N=50$ we find that $A_{0}=0.09, A_{1}=0.17, A_{2}=0.15, A_{3}=0.12, \ldots$ (approximately). For the primary and secondary correlations involving $A_{j}$ and $A_{j}^{2}$ respectively, such an insignificant difference in values hardly plays a role. But for higherorder correlations the maximum of the set $\left\{A_{j}^{\alpha_{k}}\right\}$ (still at $j=1$ ) may be orders of magnitude greater than any other element. As a result, the superposition in (43) is increasingly dominated by the term with $A_{1}^{\alpha_{k}}$, and higher-order correlations quickly converge to the first normal mode; $C_{k}(t) \approx \cos \Omega_{1} t$.

For systems of same-mass particles the set of normal mode amplitudes, given by the second equation in (14), is a periodic function of the mode index $j$ and has no single maximum. In this case the reduction of higherorder correlations to a dominating normal mode does not occur.

\section{CONCLUSION}

Temporal autocorrelation functions $\langle A(0) A(t)\rangle$ are often evaluated in the thermodynamic limit, in which case they typically decrease in a regular (non-random) fashion, either monotonically or non-monotonically. In finite systems, autocorrelation functions themselves become noisy at long time scales $t>t_{c}$; this illustrates recurrences in the dynamics of the tagged variable due to reflections of sound off boundaries. In this paper we introduced and studied some properties of the secondary correlation function $D(t)$ defined as an autocorrelation function of the primary correlation function $C(t)$. If it exists, the characteristic time of decay for $D(t)$ determines the time-scale of a typical "period" for $C(t)$, which in turn may be associated with the typical recurrence time of the targeted variable. These "typical" times may however be ill-defined mathematically (as is indeed the case for the harmonic systems discussed above), so to be more precise the secondary correlation $D(t)$ can be described as a function characterizing a distribution of recurrence 
times: for a given $t$, a larger value for $D(t)$ corresponds to a greater probability (density) that $C(t)$ will return to an assigned value in time about $t$. Comparing the secondary correlation $D(t)$ with the mean recurrence time $\tau(c)$, Eq. (6), the latter being more prevalent in literature, one notices that the two functions give complementary descriptions: while $\tau(c)$ characterizes the number of returns to an assigned value $c$, the secondary correlation $D(t)$ gives the distribution of return times regardless of the assigned value of $c$.

One interesting result is the equality $C(t)=D(t)$ for systems of same-mass particles. The equality is either exact for all $t$ or a very good approximation over the initial interval $t<t_{0}$ whose duration $t_{0}$ depends on the tagged particle's position non-monotonically. Although its derivation is quite simple, the equality of primary and secondary correlations may be a remarkable property, especially considering that the former is defined over the ensemble and the latter with time averaging. We restricted the discussion to the simplest case of one-dimensional harmonic systems, but an extension to higher dimensions appears to be straightforward. Whether the equality, or perhaps some other relation, between primary and secondary correlations still holds for nonlinear systems is an open question.

Like the primary correlation, for long time-scales the secondary correlations also develop noisy tails (see the insets in Fig. 3), which themselves can be characterized by correlations of higher order. In turn, this new tertiary function has the same structure as the secondary and primary functions, exhibiting regular decay over shorter times and fluctuating over longer times. Thus one can construct an infinite hierarchy of higher order correlations whose order-scaling properties are also interesting to study. Of course, for the particular cases when the equality $D(t)=C(t)$ holds exactly for all $t$, e.g. a harmonic chain with periodic boundary conditions, all higher order correlations are identical. We have studied higher correlations in the context of the heavy impurity problem. Here the equality of primary and secondary correlations does not hold, and the primarily correlation displays a non-random idiomatic pattern on long time scales, which becomes even more visible in correlations of higher orders. Indeed the sequence of higher-order correlations converges to the lowest normal mode.

\section{Acknowledgments}

We thank S. Shea and V. Dudnik for discussion and the anonymous referee for important suggestions.

\section{Appendix}

In this appendix we outline the derivation of expressions (3) and (14) for the momentum correlation func- tions of $i$-th particle in a harmonic chain with fixed ends, described by the Hamiltonian (1). Using the normal mode transformation

$$
q_{i}=\frac{1}{\sqrt{m}} \sum_{j=1}^{N} A_{i j} Q_{j}, \quad p_{i}=\sqrt{m} \sum_{j=1}^{N} A_{i j} P_{j}
$$

with coefficients $A_{i j}$ given by (14) and taking into account the orthogonality relation $\sum_{i=1}^{N} A_{i j} A_{i j^{\prime}}=\delta_{j j^{\prime}}$, the Hamiltonian (1) is diagonalized into the form of uncoupled normal modes

$$
H=\frac{1}{2} \sum_{j=1}^{N}\left\{P_{j}^{2}+\omega_{j}^{2} Q_{j}^{2}\right\}
$$

with frequencies $\omega_{j}$ given by (4). The normal modes are governed by the Hamiltonian equations

$$
\dot{P}_{j}=-\frac{\partial H}{\partial Q_{j}}=-\omega_{j}^{2} Q_{j}, \quad \dot{Q}_{j}=\frac{\partial H}{\partial P_{j}}=P_{j},
$$

and evolve as

$$
\begin{aligned}
P_{j}(t) & =P_{j}(0) \cos \omega_{j} t-\omega_{j} Q_{j}(0) \sin \omega_{j} t \\
Q_{j}(t) & =Q_{j}(0) \cos \omega_{j} t+\omega_{j}^{-1} P_{j}(0) \sin \omega_{j} t
\end{aligned}
$$

Assuming that initially the system is in equilibrium with canonical distribution function $\rho_{e}=Z^{-1} e^{-\beta H}$, correlations of the normal modes' initial values are $\left\langle P_{j}(0) P_{j^{\prime}}(0)\right\rangle=\delta_{j j^{\prime}} / \beta$ and $\left\langle Q_{j}(0) P_{j^{\prime}}(0)\right\rangle=0$. Then

$$
\left\langle P_{j^{\prime}}(0) P_{j}(t)\right\rangle=\left\langle P_{j^{\prime}}(0) P_{j}(0)\right\rangle \cos \omega_{j} t=\frac{\delta_{j j^{\prime}}}{\beta} \cos \omega_{j} t
$$

and the momentum correlation of the $i$-th particle is

$$
\begin{aligned}
\left\langle p_{i}(0) p_{i}(t)\right\rangle & =m \sum_{j, j^{\prime}=1}^{N} A_{i j} A_{i j^{\prime}}\left\langle P_{j^{\prime}}(0) P_{j}(t)\right\rangle \\
& =\frac{m}{\beta} \sum_{j=1}^{N} A_{i j}^{2} \cos \omega_{j} t
\end{aligned}
$$

Division of this expression by $\left\langle p_{i}^{2}(0)\right\rangle=m / \beta$ gives the normalized correlation function (14). In the case of the middle particle $i=(N+1) / 2$ (assuming $N$ is odd), $A_{i j}^{2}=$ $2 /(N+1)$ for odd $j$ and zero otherwise. In this case, one obtains the normalized correlation function in the form (3). The correlation (15) corresponding to the periodic boundary condition can be derived in a similar way. For the extension to the heavy impurity problem see e.g. [3] . 
[1] R. Zwanzig, Nonequilibrium Statistical Mechanics, Oxford University Press, New York (2001), Ch.10.

[2] P. Mazur and E. Montroll, J. Math. Phys. 1, 70-84 (1960).

[3] R. I. Cukier and P. Mazur, Physica 53, 157 (1971).

[4] M. Kac, Am. J. Math. 65, 609 (1943).

[5] J. O. Vigfusson, Physica A 98, 215 (1979).

[6] F. Jin, T. Neuhaus, K. Michielsen, S. Miyashita, M. A. Novotny, M. I. Katsnelson, and H. De Raedt, New J. Phys. 15, 033009 (2013).

[7] V. A. Benderskii and E. I. Kats, JETP 116, 1 (2013).

[8] J. Florencio and M. H. Lee, Phys. Rev. A 31, 3231 (1985).

[9] P. Hänggi and G.-L. Ingold, Chaos 15, 026105 (2005).

[10] A.V. Plyukhin and J. Schofield, Phys. Rev. E 64, 041103 (2001).

[11] H.-X. Zhou and R. Zwanzig, J. Phys. Chem. A 106, 7562
(2002).

[12] J. Rosa and M. W. Beims, Phys. Rev. E 78, 031126 (2008).

[13] Q. Wei, S. T. Smith, and R. Onofrio, Phys. Rev. E 79, 031128 (2009).

[14] H. Hasegawa, Phys. Rev. E 83, 021104 (2011); 84, 011145 (2011).

[15] A. Carcaterra and A. Akay, Phys. Rev. E 84, 011121 (2011).

[16] M. Abramowitz and I. A. Stegun, Handbook of Mathematical Functions, Dover, New York (1972).

[17] G. N. Watson, A Treatise on the Theory of Bessel Functions, Cambridge University Press, Cambridge (1995), p. 521 . 